\title{
Enjeux juridiques du contrôle des émissions personnelles de gaz à effet de serre par un dispositif de carte carbone
}

Sandrine Rousseaux, Nicolas Ochoa et Karine Foucher

\section{OpenEdition}

Journals

Édition électronique

URL : http://journals.openedition.org/developpementdurable/8732

DOI : 10.4000/developpementdurable.8732

ISSN : 1772-9971

Éditeur

Association DD\&T

Référence électronique

Sandrine Rousseaux, Nicolas Ochoa et Karine Foucher, «Enjeux juridiques du contrôle des émissions personnelles de gaz à effet de serre par un dispositif de carte carbone », Développement durable et territoires [En ligne], Vol. 2, n 1 | Mars 2011, mis en ligne le 16 février 2011, consulté le 19 avril 2019 URL : http://journals.openedition.org/developpementdurable/8732 ; DOI : 10.4000/ developpementdurable.8732

Ce document a été généré automatiquement le 19 avril 2019

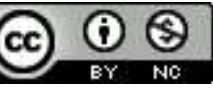

Développement Durable et Territoires est mis à disposition selon les termes de la licence Creative Commons Attribution - Pas d'Utilisation Commerciale 4.0 International. 


\title{
Enjeux juridiques du contrôle des émissions personnelles de gaz à effet de serre par un dispositif de carte carbone
}

\author{
Sandrine Rousseaux, Nicolas Ochoa et Karine Foucher
}

1 Le respect du «facteur 4 » d'ici 2050 implique une mobilisation collective de l'État, de ses administrations, des collectivités locales, des entreprises, mais aussi des particuliers, dont les actes du quotidien représentent une part importante des émissions nationales (consommation d'énergie dans l'habitat et le transport, achat de biens dont le cycle de vie est intensif en énergie) $)^{1}$.

2 La réduction des émissions personnelles de gaz à effet de serre, exprimées en équivalent dioxyde de carbone ( $\mathrm{CO}_{2}$ éq.), nécessite un changement profond des habitudes et des comportements. La nécessité de ce changement a été soulignée par le Groupe intergouvernemental d'experts sur l'évolution du climat pour la première fois dans son dernier rapport (IPPC, 2007, p. 12). Deux instruments économiques, la taxe et le système de quotas échangeables, permettent d'inciter à l'adoption de comportements sobres en carbone par l'envoi d'un signal prix. La principale différence entre ces deux instruments réside dans le choix de l'incertitude, pesant soit sur le résultat environnemental (taxe), soit sur le prix de la pollution (quotas). Un système d'échange de quotas peut être préférable lorsqu'une certitude du résultat environnemental est nécessaire (OMC, PNUE, 2009, p. 107), ce qui est le cas du changement climatique eu égard au seuil irréversible d'un réchauffement global d'environ $2^{\circ} \mathrm{C}$ par rapport aux valeurs préindustrielles.

3 L'instauration en France d'une taxe carbone est envisagée par la loi «Grenelle I » À la suite notamment de la décision du Conseil Constitutionnel rendue en décembre 2009, par laquelle il censure ses modalités de mise en place prévues par la loi de finances 2010, elle est désormais conditionnée par l'établissement d'un cadre communautaire (taxe carbone intérieure ou aux frontières), ce qui suppose l'unanimité des États membres de l'Union européenne. 
4 La taxe a été présentée comme un instrument plus efficace et moins coûteux à mettre en œuvre qu'une extension du système de quotas aux sources diffuses (Rocard, 2009, p. 5). L'extension de ce système aux émissions diffuses peut s'effectuer de deux manières : en amont, et en aval. Dans le cadre d'une extension en amont, la responsabilité des émissions pèse notamment sur les producteurs et les importateurs de combustibles fossiles. L'impact sur les particuliers est équivalent à celui d'une taxe, dans la mesure où le prix des quotas est intégré dans celui des biens qui leur sont proposés. Dans le cadre d'une extension en aval, la responsabilité des émissions pèse directement sur les particuliers. Ces derniers devraient gérer un budget carbone, fractionné en unités qui seraient débitées en fonction des émissions associées à l'achat de certains biens, et qui pourraient être échangées en fonction du solde de leur compte carbone. Cette solution semble présenter plusieurs avantages: efficacité environnementale, visibilité des émissions personnelles, contribution équitable à l'atténuation du changement climatique, libre arbitre en matière de gestion du budget carbone individuel, fiscalité progressiste (House of Commons, 2008, p. 3 ; UKERC, 2008, p. 10).

5 Le système de quotas personnels de carbone a été initialement proposé par des scientifiques au Royaume-Uni à la fin des années 1990, avant d'être pris en considération sur le plan politique. Ce dispositif novateur commence à être étudié dans d'autres pays, ainsi qu'au sein d'organisations internationales et de réseaux de collectivités locales. Il consiste à soumettre les particuliers à une obligation de réduction de leurs émissions, dont le respect est surveillé par un suivi électronique des émissions associées à leur consommation individuelle. Ce mode de contrôle des émissions personnelles soulève parfois des controverses, la carte carbone pouvant être perçue comme un instrument de rationnement potentiellement liberticide.

6 Un système de quotas d'émissions individuels, matérialisés par des crédits carbone consignés sur un compte débité par carte ou prélèvement automatique en fonction de la teneur en carbone de certains achats, et échangeables entre ceux qui ont dépassé leur budget carbone et ceux qui ne l'ont pas utilisé en totalité, n'a encore jamais été mis en place. Différentes expériences sont menées au Royaume-Uni, ou envisagées dans d'autres pays comme par exemple en Finlande (Prime Minister's Office, 2009, p. 144). Leur objectif est de tester les modalités de mise en œuvre, le fonctionnement et l'appropriation sociale de ce nouvel instrument, avant son éventuelle généralisation au niveau national. De nombreuses initiatives en matière de "carte carbone" sont par ailleurs prises depuis le début des années 2000 par le secteur privé et des autorités locales, en Europe et aux ÉtatsUnis (Rousseaux, 2009). Des banques proposent à leurs clients de compenser les émissions des biens achetés avec leur carte bancaire, par le financement de projets de développement des énergies renouvelables, de l'efficacité énergétique, et de séquestration du carbone dans la biomasse. Des cartes de fidélité visant à soutenir la consommation et les comportements durables par la collecte de points valorisables, sont également proposées par des organismes privés et des municipalités.

7 La carte carbone est donc une notion générique, qui recouvre des dispositifs pouvant obéir à des logiques différentes. Pour autant, les dispositifs existants de compensation carbone et de fidélisation « verte » n'ont pas donné lieu aux controverses soulevées par le dispositif de quotas. La question se pose alors de savoir en quoi ces trois catégories de dispositifs sont similaires ou différents, tant sur le plan de l'atténuation du changement climatique que sur celui des libertés publiques. 
Il ressort d'une analyse comparative des différents dispositifs de carte carbone que ceuxci présentent des points communs ${ }^{3}$. Leur mise en place a pour objectif ultime la protection de l'intégrité physique des personnes, puisqu'elle vise à contribuer à l'atténuation du dérèglement climatique et de son impact sur leur santé et leur sécurité. Ce mode d'atténuation du changement climatique repose sur une limitation des émissions individuelles de $\mathrm{CO}_{2}$, dont la comptabilisation nécessite un suivi de la consommation par les particuliers des produits et services couverts par les dispositifs de carte carbone. Le recueil et l'utilisation de ces informations doivent cependant préserver l'intégrité morale des personnes, et notamment le secret de leur vie privée. La fixation d'une limite aux émissions personnelles de $\mathrm{CO}_{2}$, et la gestion de données personnelles respectueuse des libertés individuelles, sont dès lors les deux principaux enjeux juridiques de l'implication des particuliers dans la lutte contre le changement climatique au moyen d'une carte carbone.

\section{La fixation d'une limite aux émissions personnelles de GES}

9 L'objectif poursuivi par les différents dispositifs de carte carbone consiste à limiter les émissions des particuliers et à soutenir financièrement la réduction de ces émissions. Le mode de régulation des émissions, ainsi que le mécanisme d'incitation économique à leur réduction, varient en fonction de la conception des dispositifs.

\subsection{Les différents modes de régulation des émissions}

10 La limitation des émissions peut s'effectuer de manière directe ou indirecte. La limitation directe consiste à plafonner ou compenser les émissions, tandis que la limitation indirecte vise à soutenir les biens et comportements les plus respectueux de l'environnement. Le principal élément de distinction entre les différents dispositifs concerne la portée de la régulation des émissions, celle-ci pouvant être impérative ou facultative.

\subsubsection{La régulation impérative}

11 Un système de quotas personnels de carbone devrait être établi à l'échelle nationale, et reposer sur une participation obligatoire des personnes âgées d'au moins 16 ou 18 ans. La quantité d'émissions de $\mathrm{CO}_{2}$ pouvant être générées par les particuliers serait déterminée par l'État, et progressivement réduite dans l'optique du « facteur 4 ».

12 Le contingent d'émissions autorisées au cours de périodes définies serait réparti entre les personnes éligibles par l'allocation de budgets carbone individuels. Chaque personne disposerait ainsi du droit d'émettre une quantité délimitée de $\mathrm{CO}_{2}$. Les budgets carbone seraient fractionnés en crédits carbone, représentatifs de l'émission d'un kilogramme de $\mathrm{CO}_{2}$ éq. ${ }^{4}$. La délivrance des crédits serait effectuée régulièrement (chaque mois ou année), et serait matérialisée par un virement sur un compte carbone. Ce compte serait débité par carte, ou par d'autres moyens, en fonction des émissions associées aux achats de produits et services couverts par le système.

13 Les émissions directes de $\mathrm{CO}_{2}$, associées à la consommation d'énergie dans l'habitat et le transport (terrestre et aérien), seraient couvertes dans un premier temps car elles sont 
les plus faciles à mesurer. Une première expérience en matière de débit par carte d'un compte carbone a été menée au Royaume-Uni en 2008. Elle portait sur les émissions liées à l'achat de carburant dans les stations-service BP. L'extension du champ d'application du système aux émissions indirectes, associées aux biens de consommation courante tels que les produits alimentaires, est plus complexe. Elle suppose d'analyser le cycle de vie de ces biens afin de déterminer leur empreinte carbone ${ }^{5}$. Des travaux de normalisation sur l'affichage carbone et environnemental des produits de consommation courante sont en cours au niveau national (PAS 2050 au Royaume-Uni, référentiel BP X30-323 en France par exemple) et international.

Les objectifs chiffrés de maîtrise des émissions personnelles peuvent également être définis par une norme. Du point de vue des particuliers, cette norme serait identique à un quota, en ce sens qu'elle les autoriserait à émettre une certaine quantité de gaz à effet de serre. Mais ce budget carbone ne serait pas fractionné en crédits pouvant être échangés sur un marché. Il serait uniquement représentatif du droit d'émettre une quantité délimitée de $\mathrm{CO}_{2}$ éq. au cours d'une période définie. Cette approche est retenue par des groupes de personnes, tels que le réseau CRAGs (Carbon Rationing Action Groups), qui se fixent des objectifs de réduction de leurs émissions, comptabilisées au moyen de calculateurs carbone.

L'allocation de budgets carbone individuels devrait induire une prise en compte de la valeur carbone des actes du quotidien et l'identification des moyens permettant de ne pas les dépasser. Le libre arbitre serait respecté, puisque les budgets carbone seraient utilisés en fonction des priorités de chacun.

La fixation d'une limite aux émissions des particuliers est cependant délicate. Elle consiste en effet à mettre fin à l'usage illimité de l'atmosphère, et à délimiter le droit d'usage de chacun. Les difficultés rencontrées en matière d'allocation des quotas aux entreprises soumises au système communautaire d'échange de quotas en attestent.

L'objectif chiffré de maitrise des émissions globales des particuliers devrait être cohérent avec les engagements nationaux et internationaux en matière de réduction des émissions de GES pris par l'État à l'horizon 2020 et 2050. Déterminé par rapport à un niveau de référence, qui pourrait correspondre aux émissions actuelles, cet objectif serait progressivement renforcé dans la perspective du « Facteur 4 ». Sa définition pourrait être confiée à un comité indépendant, afin de garantir l'effectivité environnementale du système (Fleming, 2007, p. 19).

18 La répartition des émissions autorisées entre les particuliers peut s'effectuer selon deux modalités : une allocation égalitaire - un même quota pour tous - ou une allocation en fonction des émissions historiques de chacun. L'allocation égalitaire semble préférable. Elle est plus simple et moins coûteuse à mettre en œuvre pour les pouvoirs publics (division du budget carbone global entre le nombre de participants), et plus équitable (même objectif de maîtrise des émissions à atteindre). En outre, dans la mesure où les émissions des ménages les plus aisés sont en général supérieures à la moyenne, une allocation en fonction des émissions historiques pourrait être considérée comme injuste puisqu'elle pérenniserait des situations acquises.

19 Pour autant, l'allocation d'un même quota à chaque participant ne peut être considérée comme vraiment égalitaire, le niveau des émissions personnelles dépendant de différents facteurs tels que la situation professionnelle (actif ou retraité), le lieu de résidence (rural ou urbain), les conditions climatiques, le type d'habitation (maison isolée ou 
appartement) et de chauffage, la disponibilité des transports en commun, etc. (Starkey, 2008 p. 54). La question se pose alors de savoir s'il convient d'allouer le même budget carbone à chaque personne, ou de l'ajuster afin de prendre en compte certains facteurs.

Dans l'hypothèse où un système de quotas d'émissions personnels serait établi par une législation nationale, le principe constitutionnel d'égalité ne fait pas obstacle à l'établissement de différences de traitement. Une allocation égalitaire mais ajustée est concevable, dès lors qu'elle se fonde sur des critères objectifs. Les différences de traitement doivent être en adéquation avec l'objectif de protection de l'environnement poursuivi par la mise en place du dispositif. Pourraient ainsi être pris en compte des éléments sociaux ayant un impact environnemental, comme la taille des foyers fiscaux (nombre d'enfants à charge ${ }^{6}$ ), l'éloignement contraint du domicile par rapport au lieu de travail, et le degré de maîtrise des personnes sur leur habitat. Le budget carbone individuel pourrait être initialement le même pour tous, puis augmenté par tranche en fonction de la contrainte environnementale réelle, dans un souci de justice environnementale et non sociale.

Des mesures d'accompagnement, établies parallèlement au système de quotas, peuvent cependant être nécessaires. Ces mesures pourraient être dédiées en priorité aux personnes en situation de précarité énergétique ${ }^{7}$. Elles permettraient d'intégrer des considérations de justice sociale, et faciliteraient le respect par ces personnes de leur budget carbone, tout en évitant des ajustements ou dérogations trop importants aux règles d'allocation. Ces mesures pourraient notamment consister en des interventions structurelles sur le logement, le foncier, et les transports (Theys, 2009, p. 5). Car une politique publique de limitation des émissions personnelles va inévitablement refléter les inégalités existantes en matière de revenus et d'opportunités de réduction de ces émissions (House of Commons, 2008).

\subsubsection{La régulation facultative}

Contrairement au système de quotas, les dispositifs de compensation carbone et de fidélisation «verte" reposent sur une participation volontaire des particuliers. Les premiers sont notamment proposés par des banques. Ils permettent aux particuliers de compenser l'empreinte carbone de tout ou partie de leurs achats effectués avec leur carte bancaire, par le financement de projets de réduction des émissions. Leur contribution à l'atténuation du changement climatique est cependant limitée, puisque la quantité d'émissions générées par les particuliers est inchangée. Les seconds visent à stimuler l'adoption d'actes du quotidien respectueux de l'environnement, par la délivrance et la valorisation de points lors de comportements ou d'achats durables. Mais ils ne conduisent pas à une réduction absolue des émissions car l'achat de biens durables ne dispense pas de l'achat d'autres biens qui ne le sont pas, et dont l'impact environnemental n'est pas pris en compte.

\subsection{L'incitation économique à la réduction des émissions}

23 Un dispositif de carte carbone individuelle crée une incitation économique à la réduction des émissions des particuliers. Cette incitation résulte de l'obligation de payer un prix pour les émissions générées, et/ou du droit d'obtenir une rétribution financière pour les émissions évitées. 


\subsubsection{Le paiement d'un prix pour les émissions générées}

24 Le rejet de $\mathrm{CO}_{2}$ dans l'atmosphère peut tout d'abord faire l'objet d'un paiement en monnaie carbone. Dans le cadre d'un système de quotas, les particuliers seraient soumis à une obligation juridiquement contraignante de restitution de crédits carbone pour couvrir les émissions de $\mathrm{CO}_{2}$ associées aux achats de certains biens. Les achats donneraient ainsi lieu à un double paiement, l'un en monnaie conventionnelle en fonction du prix des biens, l'autre en monnaie carbone en fonction de leur valeur environnementale. Les crédits carbone seraient prélevés du compte des particuliers, et transférés vers le compte carbone de l'autorité en charge de leur allocation ou celui du fournisseur de biens, en fonction de la conception du dispositif.

Les émissions de $\mathrm{CO}_{2}$ peuvent aussi être payées en monnaie conventionnelle. Cette modalité s'appliquerait en cas d'allocation payante des crédits carbone aux particuliers (Blake, 2008, p. 137), bien que l'allocation gratuite soit le plus souvent envisagée. Elle s'appliquerait également en cas d'oubli ou de non détention d'une carte, par exemple par les mineurs et les résidents temporaires qui ne seraient pas soumis à un dispositif similaire dans l'État dont ils relèvent. La procédure de restitution des crédits serait prise en charge par le vendeur, qui les achèterait sur le marché du carbone et intégrerait leur prix dans celui des biens achetés. Dénommée "pay as you go ", cette procédure peut s'assimiler à une taxe sur le carbone ajouté. Un prix devrait enfin être payé en cas de dépassement de l'objectif de réduction des émissions imparti aux particuliers. Une pénalité pour émissions excédentaires pourrait s'appliquer en cas de non-respect de la norme énonçant cet objectif, ou de dépassement de la dotation de crédits carbone. Dans ce second cas, des crédits supplémentaires devraient être acquis sur le marché, soit par les particuliers ou les gestionnaires de leur compte (réapprovisionnement du compte carbone), soit par le vendeur (procédure « pay as you go »).

Les émissions font également l'objet d'un paiement en monnaie conventionnelle dans le cadre des dispositifs de compensation proposés par des banques. Des crédits carbone sont achetés sur le marché pour couvrir les émissions associées aux achats des porteurs de carte bancaire. L'incitation au changement des comportements est cependant faible, voire inexistante, puisque les émissions générées sont annulées par des réductions réalisées par ailleurs. Ce risque de dédouanement est renforcé par le fait que les crédits carbone sont le plus souvent achetés non pas par les particuliers, mais par les banques.

\subsubsection{La rétribution financière pour les émissions évitées}

27 Le droit d'émettre du $\mathrm{CO}_{2}$ octroyé aux participants à un système de quotas personnels constituerait un droit acquis. Les crédits carbone n'ayant pas été utilisés du fait de l'adoption d'actes du quotidien durables pourraient être épargnés ou cédés à d'autres personnes, à titre onéreux ou gratuit. Dans l'éventualité où l'objectif de maîtrise des émissions serait énoncé par une norme, les réductions supplémentaires pourraient également être valorisées financièrement par l'octroi d'une récompense. Ces réductions d'émissions ne seraient cependant pas cessibles, et ne pourraient pas être épargnées. La valorisation des émissions évitées peut être neutre sur le plan financier, en fonction de la conception du mécanisme de bonus-malus.

Les dispositifs de soutien à la consommation et aux comportements durables prévoient eux aussi une rétribution financière, par l'attribution de points pour des comportements 
ou actes d'achat spécifiques. Les points sont valorisés sous la forme de cadeaux ou de réductions à l'achat de certains produits et services. Ces derniers ne sont pas toujours des biens respectueux de l'environnement, la valorisation des points pouvant varier selon les programmes.

\section{La gestion de données personnelles respectueuse des droits fondamentaux}

L'image de «Big Brother", couplée à l'évocation populaire des «libertés », effraie instinctivement en suscitant des images collectives et personnelles d'aliénation, de viol de son intimité. La question de savoir si un dispositif de carte carbone présente des aspects liberticides impose au préalable de définir les droits et libertés susceptibles d'être atteints, et de les mettre en balance avec ceux que le dispositif cherche à garantir. Cette question revient à confronter la limitation des atteintes à l'intégrité physique des personnes liées au dérèglement climatique, objectif ultime poursuivi par le dispositif, avec la préservation de l'intégrité morale de ces mêmes personnes, et plus particulièrement du droit de la protection de leurs données personnelles. Elle doit ainsi être replacée dans le contexte plus général de la protection des droits fondamentaux, dont le but est de préserver les intérêts privés fondamentaux des personnes physiques.

La protection de l'intégrité physique des personnes occupe en droit la première place de la hiérarchie substantielle des intérêts fondamentaux. Pour autant, cet objectif ne prime sur la préservation de l'intégrité morale que si la conciliation opérée entre les deux intérêts répond aux principes de nécessité et de proportionnalité. Ces deux principes s'imposent à tous les juges français et internationaux lorsqu'ils doivent concilier sur un point précis des droits fondamentaux distincts et potentiellement en conflit. Dès lors, la carte carbone ne pourra légalement porter atteinte à des droits fondamentaux que si cette atteinte est non seulement nécessaire pour atteindre un objectif supérieur à celui de la protection des données personnelles, mais aussi proportionnée à la réalisation de cet objectif.

31 Les enjeux du débat étant précisés sur le plan théorique, quelles sont les conséquences concrètes d'un dispositif de carte carbone en termes d'atteinte aux libertés ? Cela suppose d'analyser les conditions de recueil, de stockage et d'utilisation des données personnelles.

\subsection{Le recueil de données personnelles}

32 Des données personnelles sont collectées lors de l'inscription des particuliers aux dispositifs de carte individuelle, établis par des organismes privés ou des autorités publiques. Elles comprennent des informations permettant de vérifier l'identité des personnes et sont consignées dans un fichier central. Le recueil de données sur la consommation individuelle vise quant à lui à comptabiliser la quantité d'émissions générées ou la quantité de points de fidélité collectée.

\subsubsection{La nature des données recueillies}

33 La loi informatique et libertés du 6 janvier 1978 instaure un régime de droit commun concernant les données personnelles ${ }^{8}$, et un régime de protection renforcée pour les données dites sensibles (relatives notamment aux opinions philosophiques ou religieuses, 
à la santé ou à la sexualité des personnes). Les émissions de $\mathrm{CO}_{2}$, ainsi que les points de fidélité, se présentent a priori comme des données personnelles de droit commun. Il en résulte une application du régime normal des garanties prévues par la loi informatique et libertés aux dispositifs de carte carbone, ainsi qu'un contrôle subséquent de la CNIL (Commission Nationale Informatique et Libertés) ${ }^{9}$.

Les émissions de $\mathrm{CO}_{2}$ et les points de fidélité peuvent-ils constituer des données sensibles? Cela semble fort improbable si la carte carbone ne retient que le score environnemental attribué à chaque acte de consommation, sans garder de trace du bien consommé en lui-même. Dans ces conditions d'anonymisation des biens consommés, le niveau normal de protection prévu par la loi informatique et libertés est applicable. Cette protection comprend tout d'abord des garanties de type institutionnel, avec un contrôle de la CNIL pouvant intervenir a priori et a posteriori ${ }^{10}$. Dans l'éventualité où un dispositif national de quotas personnels reposant sur une participation obligatoire serait mis en place par le législateur, l'avis de la CNIL, qui dispose de pouvoirs consultatifs sur les projets de loi ou de décret ayant trait à la protection des données personnelles, serait publié au Journal officiel le même jour que la norme instaurant le dispositif. Chacun pourrait ainsi prendre connaissance des aspects liberticides ou non du dispositif instauré. Les personnes dont des données font l'objet d'un recueil et d'un traitement bénéficient également de garanties individuelles, telles que le droit de consentir ou pas au recueil et au traitement de ses données personnelles (auquel cas la procédure "pay as you go » préalablement mentionnée serait applicable), et le droit d'obtenir copie des informations personnelles contenues dans le traitement.

L'établissement d'un dispositif obligatoire de carte carbone reposant sur l'allocation de quotas d'émission personnels serait soumise à l'obtention d'une autorisation de la CNIL, qui ne pourrait être délivrée qu'après vérification du respect de la loi informatique et libertés. La procédure de déclaration, applicable aux dispositifs facultatifs tels que les cartes de compensation carbone et cartes de fidélisation verte, permet également à la CNIL d'examiner les conditions de traitement des données personnelles.

\subsubsection{Les modalités de recueil des données} valeur environnementale des actes couverts s'effectue de façon automatique au moyen d'une carte. Il devrait en aller de même dans le cadre d'un dispositif de quotas. Une déclaration manuelle par les participants de la teneur en carbone indiquée sur les relevés d'achat est concevable. Mais cette procédure serait fastidieuse et pourrait donner lieu à des fraudes, ce qui porterait atteinte à l'effectivité du dispositif. Les émissions seraient mesurées au moyen de technologies de l'information et de la communication (TIC), telles que des cartes, mais aussi des téléphones portables ou des compteurs intelligents.

Les données ainsi recueillies feraient l'objet d'une transmission automatisée à la base de données comprenant les comptes carbone des participants aux dispositifs, où elles seraient consignées, de la même manière que dans le cadre des dispositifs de carte existants.

Seule l'utilisation d'instruments de paiement électronique pourrait permettre de déclarer les émissions et de déclencher la procédure de débit automatique des comptes carbone. La question du caractère potentiellement liberticide d'une société dans laquelle la valeur environnementale du moindre acte d'achat est systématiquement tracée et consignée 
pourrait être évoquée à mesure de l'extension progressive du champ d'application du système de quotas. L'utilisation de la monnaie fiduciaire et scripturale, n'impliquant pas de traitement automatisé de données sur les émissions personnelles de $\mathrm{CO}_{2}$, ne serait toutefois pas interdite. Le prix du carbone serait dans ce cas intégré à celui des biens, selon la procédure "pay as you go». Un surcoût serait cependant supporté pour les particuliers ne souhaitant pas être soumis aux obligations en matière de comptabilisation des émissions et de restitution des crédits carbone. Les crédits seraient achetés par le vendeur des biens au prix prévalant sur le marché du carbone, auquel s'ajouteraient des frais de gestion dus aux intermédiaires de marché.

\subsection{Le stockage des données personnelles}

39 Deux sortes d'obligation pèsent sur l'auteur du traitement de données personnelles consignées : celle de ne conserver les données recueillies que pour une durée nécessaire à la finalité du traitement, et celle d'assurer la sécurité matérielle et donc la confidentialité des données stockées.

\subsubsection{La durée de conservation des données}

La loi informatique et libertés (article 6.5) et le code pénal (article 226-20) énoncent l'obligation de respect d'une durée de conservation proportionnelle à la finalité du traitement. Les données doivent ensuite être supprimées ou rendues anonymes.

41 La finalité des dispositifs de carte carbone consiste en une gestion de données comptables, relatives à la quantité d'émissions générées ou à la quantité de points collectés. Des mouvements sur des comptes bancaires peuvent en résulter. Des comptes approvisionnés en monnaie conventionnelle sont débités lors de l'achat de crédits carbone (dispositifs de compensation et de quotas), et en cas de dépassement des budgets carbone alloués (dispositif de quotas). Des comptes approvisionnés en monnaie carbone pourraient également être débités lors de l'achat de biens (dispositif de quotas).

Les données relatives aux émissions et aux transferts de monnaie carbone seraient conservées au cours des périodes pluriannuelles de mise en œuvre du système de quotas, à l'instar des systèmes existants applicables à certains États et activités économiques. La durée de conservation des données relatives à la monnaie carbone serait ainsi inférieure à celle relative à la monnaie conventionnelle.

\subsubsection{La sécurité des données conservées}

43 L'introduction dans un système informatisé est un délit ${ }^{11}$. Ce risque est limité dans le cadre des programmes de fidélité, dans la mesure où le préjudice financier lié à la valorisation de points obtenus de manière illégale est relativement faible, tant pour les particuliers que pour les développeurs des programmes. Le risque est plus important dans le cadre de dispositifs d'allocation de budgets carbone, dans la mesure où les particuliers seraient soumis à une obligation de restitution de crédits dont la valeur monétaire devrait croître à mesure du renforcement de leur rareté.

L'obligation de sécurisation du système informatique, pesant sur les auteurs de traitement informatisé de données personnelles, s'appliquerait naturellement aux bases de données comprenant les comptes carbone. Il est envisagé de créer une banque 
gouvernementale du carbone, ou d'utiliser les infrastructures bancaires existantes, cette seconde option étant moins coûteuse (Roberts et Thumim, 2006 ; Lane et al., 2008). Les comptes carbone des particuliers pourraient également être ouverts dans les registres nationaux créés en application du Protocole de Kyoto, dans l'éventualité où les crédits alloués aux particuliers seraient des actifs carbone des États.

\subsection{L'utilisation croisée avec d'autres données personnelles}

Deux risques peuvent se poser en termes d'atteinte aux droits fondamentaux. Le premier concerne le détournement de finalité d'un traitement de données personnelles. Le second a trait à l'interconnexion entre le fichier recensant les émissions individuelles et d'autres fichiers à finalité non déterminée.

\subsubsection{Le détournement de finalité}

Tout traitement de données personnelles n'est licite que dans la mesure où il est nécessaire à l'accomplissement d'une finalité légitime ${ }^{12}$, ce qui est le cas pour les dispositifs de carte carbone dont l'objet est de lutter contre le changement climatique et de protéger in fine l'intégrité physique de la personne humaine. Le respect de cette obligation faisant l'objet d'un contrôle préventif, tout détournement de finalité serait déclaré illégal lors de l'examen préalable du dispositif par la CNIL. Si un détournement de finalité venait à être constaté dans la pratique ultérieure, il serait sanctionné en application de l'article 226-21 du code pénal ${ }^{13}$.

\subsubsection{L'interconnexion}

Deux sous-questions distinctes se posent, à savoir l'interconnexion à raison du croisement des fichiers de données ou à raison du support matériel employé pour la carte carbone.

L'interconnexion volontaire des fichiers n'est autorisée au titre de la loi informatique et libertés que sous réserve de nécessité absolue. Le potentiel liberticide d'un croisement de fichiers résulte de la plus-value en termes d'informations personnelles révélées par l'association de données distinctes, pouvant favoriser la constitution de profils informatisés. Ceci a notamment conduit la Cour européenne des droits de l'homme à considérer que dans certains cas, le croisement de données personnelles ne pouvant être considérées comme sensibles du seul fait de leur nature équivalait, du fait de la plus-value informationnelle liée à l'interconnexion, à un traitement de données sensibles ${ }^{14}$. La sensibilité d'une donnée personnelle dépend donc autant de sa nature que du traitement auquel elle a été soumise. Or c'est précisément ce genre de traitement de données qui présente des risques en termes d'atteintes aux droits fondamentaux des personnes.

Dans ces conditions, si les données concernant les émissions personnelles de $\mathrm{CO}_{2}$ éq. ne peuvent être considérées comme des données sensibles par nature, elles peuvent théoriquement acquérir une telle sensibilité par agrégation, en étant recoupées avec d'autres données personnelles. Mais même dans ce cas, une interconnexion ne présente que peu voire pas d'enjeux en termes de risque d'atteinte aux droits fondamentaux. Le recoupement des émissions personnelles avec d'autres données nominatives ne révèle rien de profondément intime, si ce n'est l'empreinte carbone des personnes. Cette donnée peut s'apparenter à celle relative à la consommation d'un bien unique comme l'eau ou 
l'électricité, ce qui ne constitue pas une donnée sensible. Le potentiel liberticide est donc très faible du fait de la teneur informationnelle limitée de ces données.

Ces informations étant toutefois des données personnelles, elles sont soumises à un régime légal strict. Leur traitement ne peut intervenir qu'après avoir respecté des règles minimales, comme celle de justifier que le traitement est légal, nécessaire pour la réalisation d'une finalité supérieure, et strictement proportionné à cette finalité. Ainsi, il n'est pas permis par la loi de procéder à des « vérifications de routine » discrétionnaires sous un prétexte fallacieux, car toute information personnelle est revêtue a priori et par principe d'un voile légal de secret par la Loi informatique et libertés.

51 S'agissant du support matériel employé pour le suivi de la consommation individuelle des biens couverts par les dispositifs de carte carbone, il peut s'agir d'une carte spécifique (nominative ou non), ou de TIC multifonction telles qu'un téléphone portable faisant office d'instrument de paiement ou une carte dotée d'une puce permettant plusieurs traitements avec des finalités distinctes. Les programmes existants de carte carbone ont d'ailleurs intégré une fonctionnalité de comptabilisation du carbone dans une carte de fidélité, une carte bancaire, ou une carte d'identité électronique. Une carte multifonction présente un avantage en termes de praticité pour l'utilisateur, puisqu'elle dispense de se munir d'une carte supplémentaire. Une carte bancaire dotée d'une fonctionnalité carbone par exemple permet la mesure et la déclaration simultanées des émissions et la réalisation d'opérations simultanées sur deux comptes approvisionnés en monnaie différente. Un support technique commun à plusieurs fonctions ne crée pas forcément en soi une interconnexion au sens de la loi. Mais il la favorise indéniablement, même si des solutions techniques existent pour permettre une certaine étanchéité entre les deux fonctions distinctes que sont le débit/crédit de monnaie conventionnelle et parallèle.

\section{Conclusion}

52 Une analyse comparative des différents dispositifs de carte carbone individuelle, existants ou en projet, permet de constater que les controverses soulevées au sujet de leurs potentialités liberticides et totalitaires ne sont pas réellement fondées.

Certes des atteintes à l'intégrité morale des personnes peuvent être autorisées en droit, mais si et seulement si elles ont pour objet le respect d'un droit fondamental plus important - la protection de l'intégrité physique des personnes en l'espèce. L'arsenal juridique existant en matière de protection des données personnelles comporte cependant des garanties suffisantes pour prévenir tout risque d'atteinte à l'intégrité morale des personnes lié à la mise en place d'une carte carbone. Il peut d'ailleurs être observé que la gestion de données personnelles est intégrée dans les dispositifs existants de carte de compensation carbone et de fidélisation verte, sans que soit évoquée la virtualité liberticide de ces derniers. Ces dispositifs volontaires ont pourtant le même effet qu'un système de quotas d'émission sur le plan des droits fondamentaux, même si leur impact est moindre en raison du nombre limité de participants. Surtout, un système de quotas personnels vise à instaurer un suivi des émissions de $\mathrm{CO}_{2}$ associées aux achats de certains biens, et non pas des achats eux-mêmes. Ces données personnelles ne sont pas des données sensibles, en ce sens qu'elles ne révèlent rien de profondément intime sur les particuliers si ce n'est leur empreinte carbone. 

émissions personnelles, à la différence d'un système de quotas. Seul un dispositif national d'allocation de budgets carbone reposant sur une participation obligatoire des particuliers, établi parallèlement à d'autres dispositifs applicables aux organisations (entreprises, administrations, collectivités locales) ou couvrant l'économie nationale, semble toutefois être doté d'une efficacité environnementale suffisante pour parvenir graduellement au «facteur 4 » d'ici 2050 , objectif énoncé au niveau législatif ${ }^{15}$. Il en résulterait un partage de la responsabilité des émissions entre les particuliers et les organisations. Se pose ainsi la question de l'acceptation d'une politique publique destinée à limiter les émissions diffuses, telle que récemment posée lors des débats relatifs à l'instauration d'une taxe carbone en France. par les quantités. La notion de rationnement ne semble cependant pas appropriée car elle renvoie à l'idée d'une limitation fixe des émissions. Les termes de limite ou de plafond flexible sont plus opportuns. Le budget carbone individuel serait en effet modulable, puisqu'il serait assorti d'un mécanisme de récompense et de pénalité en fonction de son respect ou de son dépassement.

Le dispositif de quotas personnels de carbone devrait constituer une option sérieuse lorsque les politiques existantes seront considérées comme n'étant pas en mesure de réduire suffisamment les émissions des particuliers (Bird, Lockwood, 2009, p. 46). Un récent état de l'art de la science sur le climat indique que le changement climatique s'accélère à un rythme plus rapide que prévu, et que certains points de non-retour conduisant à des perturbations irréversibles des principaux écosystèmes et éléments du système Terre sont déjà franchis (UNEP, 2009, p. ii). La nécessité d'une réduction rapide et substantielle des émissions de gaz à effet de serre est à nouveau soulignée.

\section{BIBLIOGRAPHIE}

Bird J., Lockwood M., 2009, Plan B? The prospects for personal carbon trading, Institute for Public Policy Research, ISBN 9781860303289, London, 54 p.

Blake A., 2008, Country carbon rationing, 5 p., First international conference on Economic Degrowth for Ecological Sustainability and Social Equity, Paris, http://events.it-sudparis.eu/ degrowthconference.

Department for Environment, Food and Rural Affairs, 2008, Synthesis report of the findings from Defra's pre-feasibility study into personal carbon trading, London, $26 \mathrm{p}$.

Fleming D., 2007, Energy and the common purpose. Descending the energy staircase with tradable energy quotas (TEQS), The lean economy connection, 3ème éd., ISBN 0-9550849-1-1, London, 26 p.

House of Commons, Environmental Audit Committee, 2008, Personal Carbon Trading, Fifth report of Session 2007-08, London, $164 \mathrm{p}$.

Développement durable et territoires, Vol. 2, n 1 | Mars 2011 
Intergovernmental Panel on Climate Change, 2007, Summary for Policymakers, Climate Change 2007 : Mitigation. Contribution of Working Group III to the Fourth Assessment Report, Cambridge and New York, Cambridge University Press, 23 p.

Lane C., Harris B., Roberts S., 2008, An analysis of the technical feasibility and potential cost of a personal carbon trading scheme. A report to the Department for Environment, Food and Rural Affairs, Accenture \& Centre for Sustainable Energy, London, 46 p.

Organisation Mondiale du Commerce et Programme des Nations-Unies pour l'Environnement, 2009, Commerce et changement climatique, ISBN 978-92-870-3523-3, Genève, OMC, 212 p.

Prescott M., 2008, A persuasive climate. Personal trading and changing lifestyles, RSA, London, $31 \mathrm{p}$.

Prime Minister's Office, 2009, Government foresight report on long-term climate and energy policy: towards a low-carbon Finland, Prime Minister's Office Publications, ISBN 978-952-5807-70-7, Helsinki, $188 \mathrm{p}$.

Roberts S., Thumim J., 2006, A rough guide to individual carbon trading. The ideas, the issues and the next steps, Report to Defra, Centre for sustainable energy, London, $41 \mathrm{p}$.

Rocard M., 2009, Rapport de la Conférence des experts et de la table ronde sur la contribution climat et énergie, Paris, $84 \mathrm{p}$.

Rousseaux S., 2009, État des lieux international des programmes de 'carte carbone' pour les particuliers (Europe et États-Unis), Nantes, Rapport pour l'ADEME, 69 p., http://hal.archives-ouvertes.fr/ hal-00368067/fr.

Starkey R., 2008, Allocating emission rights: are equal shares, fair shares?, Working paper 118, University of Manchester, Tyndall Centre for Climate Change Research, 74 p.

Szuba M., Semal L., 2010, « Rationnement volontaire contre "abondance dévastatrice" : l'exemple des Carbon Rationing Action Group », Sociologies pratiques, n²0, 7 p.

Theys J., 2009, Vers des villes post-carbone. Six sentiers de transition, Horizons 2030-2050, $\mathrm{n}^{\circ} 2$, Commissariat général au développement durable, MEEDDM, Paris, $10 \mathrm{p}$.

UK Energy Research Centre, 2008, Personal Carbon Trading (PCT), Bringing together the research community. Workshop Report, UKERC/MR/MP/2009/001, Oxford, 48 p.

United Nations Environment Programme, 2009, Climate Change Compendium, ISBN

978-92-807-3034-0, Nairobi, Kenya, 76 p.

\section{NOTES}

1. « $50 \%$ des émissions de $\mathrm{CO}_{2}$ sont le fait des ménages en France. Chaque Français émet aujourd'hui du fait de ses activités quotidiennes, environ 10 tonnes équivalent $\mathrm{CO}_{2}$ par an. Or, pour prétendre à un mode de vie durable, chaque citoyen devrait diviser par 4 ses émissions afin de ne pas dépasser un plafond annuel de 2350 kilos équivalent $\mathrm{CO}_{2}$ ", Agence de l'environnement et de la maîtrise de l'énergie (2008), Dossier éco-consommation.

2. Article 2.II de la loi $n^{\circ} 2009-967$ du 3 août 2009 de programmation relative à la mise en œuvre du Grenelle de l'environnement, JORF 5 août 2009.

3. Cet article ne porte pas sur les dispositifs qui consistent à compenser ou délivrer une quantité forfaitaire d'émissions ou de points de fidélité en fonction de l'utilisation d'une carte bancaire (nombre d'opérations mensuelles ou montant des dépenses effectuées). 
4. Les crédits actuellement en circulation sur les marchés du carbone sont représentatifs de l'émission d'une tonne d'équivalent $\mathrm{CO}_{2}$.

5. La comptabilisation des émissions personnelles peut conduire à intégrer les émissions générées en dehors du territoire national. Cette approche est différente des inventaires d'émission de GES réalisés au titre de la Convention-cadre des Nations Unies sur les changements climatiques, ceux-ci ne prenant en compte que la production nationale d'émissions.

6. Les recherches menées au Royaume-Uni envisagent l'allocation d'une quantité de crédits équivalente à la moitié ou les deux tiers d'un quota par enfant. Dans une réponse au rapport sur les quotas personnels de carbone réalisé par le Comité d'audit environnemental de la Chambre des Communes, le gouvernement a indiqué en novembre 2008 que l'allocation d'un tiers de quota supplémentaire par enfant représente un compromis raisonnable.

7. «Les marges de manœuvre des personnes les moins favorisées sont souvent restreintes, notamment en ce qui concerne les dépenses énergétiques (chauffage et transport principalement)», D. Cohen (2009): Sortie de crise. Vers l'émergence de nouveaux modèles de croissance?, Centre d'Analyse Stratégique, Paris, p. 84

8. Les données personnelles sont définies par l'article 2 alinéa 2 comme "toute information relative à une personne physique identifiée ou qui peut être identifiée, directement ou indirectement, par référence à un numéro d'identification ou à un ou plusieurs éléments qui lui sont propres. Pour déterminer si une personne est identifiable, il convient de considérer l'ensemble des moyens en vue de permettre son identification dont dispose ou auxquels peut avoir accès le responsable du traitement ou toute autre personne ».

9. Le contrôle exercé par la CNIL n'est pas le seul, mais c'est de loin le plus médiatique. La Cour européenne des droits de l'homme et la Cour de justice de l'Union Européenne exercent également un contrôle rigoureux sur le respect par les États membres des règles internationales en matière de protection des données personnelles, y compris pour des traitements de données survenus dans des domaines «régaliens" comme la police ou les impôts. Ces règles sont énoncées au niveau européen par la Convention pour la protection des personnes à l'égard du traitement automatisé des données à caractère personnel (STE n 108) du 28 janvier 1981 (JORF 20 nov. 1985), et au niveau communautaire avec la directive 95/46/CE du Parlement européen et du Conseil du 24 octobre 1995, relative à la protection des personnes physiques à l'égard du traitement des données à caractère personnel et à la libre circulation de ces données (JOUE $\mathrm{n}^{\circ} \mathrm{L}$ 281, 23 nov. 1995 ).

10. La CNIL dispose depuis la réforme de la loi informatique et libertés du 6 août 2004 de pouvoirs de sanction pécuniaire extrêmement dissuasifs, ainsi que de pouvoirs d'enquête et de contrôle extrêmement complets.

11. L'article 323-1 du Code pénal dispose que «[1]e fait d'accéder ou de se maintenir, frauduleusement, dans tout ou partie d'un système de traitement automatisé de données est puni de deux ans d'emprisonnement et de 30000 euros d'amende. Lorsqu'il en est résulté soit la suppression ou la modification de données contenues dans le système, soit une altération du fonctionnement de ce système, la peine est de trois ans d'emprisonnement et de 45000 euros d'amende».

12. Aux termes de l'article 6.3 de la loi informatique et libertés, les données recueillies doivent être « adéquates, pertinentes et non excessives au regard des finalités pour lesquelles elles sont collectées et de leurs traitements ultérieurs ".

13. «Le fait, par toute personne détentrice de données à caractère personnel à l'occasion de leur enregistrement, de leur classement, de leur transmission ou de toute autre forme de traitement, de détourner ces informations de leur finalité telle que définie par la disposition législative, l'acte réglementaire ou la décision de la Commission nationale de l'informatique et des libertés autorisant le traitement automatisé, ou par les déclarations préalables à la mise en œuvre de ce traitement, est puni de cinq ans d'emprisonnement et de 300000 Euros d'amende». 
14. CEDH 4 mai 2000, req. $n^{\circ} 28341 / 95$, Rotaru c/ Roumanie, rec. 2000-V. Dans un tel cas, le régime renforcé de protection des données sensibles s'applique donc a posteriori.

15. Loi de programme fixant les orientations de la politique énergétique, 13 juillet 2005 (JORF 14 juillet) et loi « Grenelle I » du 3 août 2009 (JORF 5 août).

\section{RÉSUMÉS}

La carte carbone individuelle est un dispositif innovant, qui permet d'impliquer les particuliers dans la lutte face au changement climatique. Ce dispositif consiste à limiter leurs émissions de gaz à effet de serre, ce qui suppose de suivre leur consommation de certains produits et services. Ce mode de contrôle des émissions personnelles soulève parfois des controverses, la carte carbone pouvant être perçue comme un instrument de rationnement potentiellement liberticide. Ces deux aspects sont discutés sur la base d'une analyse comparative de la conception et des modalités de mise en œuvre des différents dispositifs de carte carbone, établis ou envisagés en Europe et aux États-Unis. Il en ressort que les controverses ne sont pas réellement fondées.

The individual carbon card is an innovative mechanism that helps involve individuals in the fight against climate change. It consists in limiting their greenhouse gas emissions, which requires monitoring a part of their goods and services consumption. This method of personal emissions control is much debated since the carbon card may be perceived as a rationing tool and raises civil liberty issues. Both of these controversial aspects are discussed on the basis of a comparative analysis of how the various carbon card programs, established or contemplated in Europe and in the USA, are designed and implemented. The findings are these controversies are somewhat groundless.

\section{INDEX}

Keywords : carbon card, climate change, individuals, personal allowances, rationing, civil liberty Mots-clés : budget carbone individuel, carte carbone, changement climatique, libertés individuelles, particuliers, quotas personnels, rationnement

\section{AUTEURS}

\section{SANDRINE ROUSSEAUX}

Sandrine Rousseaux est chargée de recherche au CNRS, au laboratoire Droit et Changement Social à Nantes. Ses travaux en droit de l'environnement portent principalement sur le changement climatique.

\section{NICOLAS OCHOA}

Nicolas Ochoa est doctorant en droit à l'Université Paris I, et chargé d'enseignement à l'Université de Nantes. Il prépare une thèse sur le sujet : « La protection des données personnelles à l'épreuve des NTIC». 


\section{KARINE FOUCHER}

Karine Foucher est maître de conférences de droit public à l'Université de Nantes. Ses travaux portent sur le principe de précaution, et sur les liens entre droit constitutionnel et environnement. 